\title{
Minimum Power Consuming Algorithm to Select Relay Node of Mimo Integrated Mobile Ad Hoc Network
}

\author{
Swati Chowdhuri ${ }^{*}$, Pranab Banerjee ${ }^{2}$, Sheli Sinha Chaudhuri and Dibyendu Mal ${ }^{3}$ \\ 'Department of Electronics and Communication Engineering, Techno India, Batanagar, Kolkata - 700141, \\ West Bengal, India; swati.chowdhuri@gmail.com \\ ${ }^{2}$ Department of Electronics and Tele Communication Engineering, Jadavpur University, Kolkata - 700032, \\ West Bengal, India; Pkbju65@gmail.com, shelism@rediffmail.com \\ ${ }^{3}$ Department of Physics, Heritage Institute of Technology, Kolkata - 700107, \\ West Bengal, India; dipphysics@gmail.com
}

\begin{abstract}
Objectives: To design an MIMO integrated Mobile Ad Hoc Network (MANET) in an energy efficient way. As MANETs are battery operated, so energy constrained is an important factor to design this integrated network. It is very essential to develop a routing algorithm using energy awareness consideration. Methods/Statistical Analysis: A Minimum Power Consumed Routing (MPCR) algorithm is developed which is an integration of cooperative transmission process. Most of existing routing algorithm of Mobile Ad Hoc Network is based on distance and metric value, but this proposed algorithm is based on effective received power by the mobile nodes. Minimum effective power consumes minimum energy for transmission. The route selection of this algorithm is simulated in MATLAB environment and comparative study is done of this algorithm with some other existing algorithm. Findings: This algorithm selects relay node and supports short distance communication which can improve the Signal to Noise Interference Ratio (SNIR). The performance analysis of proposed routing algorithm improves Signal to Noise Interference Ratio (SNIR) resulting in better cooperative transmission. Novelty/ Applications: This algorithm suitably works on any hazardous environment where structured network implementation is not possible.
\end{abstract}

\section{Keywords: Algorithm, MANET, MIMO, Minimum Power, Relay Node}

\section{Introduction}

Advancement in communication system enables MANET to broadcast information packets in any hazardous environment. MIMO technology with multiple antenna elements serves a number of users' equipment simultaneously which share mutual resources. One of the efficient methods of transmitting packets from source to destination receiver node in MANET is broadcasting and designing an efficient broadcasting protocol is the most demanding task in MIMO integrated Mobile Ad Hoc Networks. Mobility, dynamic topology and resource sharing are the unique characteristics of MIMO integrated MANET. The mobility of these nodes changes the network topology dynamically causing frequent path failures which is the limitation of conventional flooding method $^{1}$. Packet broadcasting of MIMO implemented MANET is mainly done in resource sharing mechanism. Limited radio range of mobile nodes necessitate multihop transmission in the integrated network to establish communication and packets transmitted from the valid mobile source node may not reach the target node in a single hop but by multiple hops through some intermediate node called relay node. Selection of intermediate node is an important criterion as these nodes use the valuable resources of the network like battery power and bandwidth etc ${ }^{2}$. Packet forwarding to the entire mobile

${ }^{*}$ Author for correspondence 
network, paging of a node, network management, route discovery and overhead control has been served by this broadcasting method 3.4 . Different broadcasting technique has been proposed and studied by many researchers of which performance analysis of conventional routing protocol is done $e^{\underline{5}}$ considering single hop and multi hop routing. Author ${ }^{\underline{6}}$ compared the performance of protocols in NS-2 simulation environment and indicated improvement. Discrete optimal sizing of truss using adaptive directional differential evolution and simulation of small link conventional routing in OPNET environment has been done ${ }^{7,8}$. Comparison of performance of single-hop and multi-hop routing followed by simulation has been done for ordinary ad hoc network $\frac{210}{2}$. Topology and geometry optimization using ECBO is done $\mathrm{e}^{\underline{11}}$. A hybrid routing protocol is analyzed where distance, metric value and node failure has been considered $\frac{12,13}{}$. Selection of relay node for routing process based on coding cooperative approach and real time channel state approach has been described $\frac{14,15}{}$. In coding cooperative approach, authors describe the basic idea that each user must transmit increasing redundant information for cooperative partner and the basic principle of real time channel state approach is to transmit real time channel information of source node to relay node and relay to destination node. The best partner selection algorithm using stable matching concept has been discussed $\frac{16}{}$. Here authors focused on 'selective' decode-forward transmission where relay station only decodes the data and retransmit the data to the destination. From the review work we find most of the routing algorithm has been developed on basis of distance, metric value and node failure. Development of this proposed algorithm is based on selection of best suitable relay node. The best suitable relay nodes selection process is done considering the parameters propagation loss and probability of collision during transmission, which gives most effective transmission power. This effective transmission power makes up the proposed hybrid routing algorithm in different terrain presented in this paper. We propose a routing protocol considering all these parameters in an energy efficient way.

The rest of this paper is organized as follows. In Section 2 defined rationality of relay node. Section 3 is described proposed relay node selection method followed by supported MPCR algorithm in Section 4. Section 5 shows simulation results and discussion. Finally, Section 6 summarizes the main findings of this work.

\section{Rationality of Relay Node}

Conventional flooding mechanism has some limitation due to the limited power of mobile nodes, more interference and fading effect. A pragmatic alternative will be to replace a single long range link by a chain of short range links connecting series of intermediate nodes between source and destination. Optimized value of short distance is considered to minimize the power consumption such that relay node placed within the transmission range of the transmitting node. $S N I R=\frac{P}{r I}$ where $\mathrm{P}$ is transmitting power, $r$ is distance between two communicating nodes and I is interference ${ }^{17}$. The intermediate node receives the signal from the source, processes and retransmits to the next node of the chain. The intermediate nodes are known as relay node and by multiple hoping through relay nodes, the packet reaches the destination node. Relay node provides seamless communication even if a node becomes partially inefficient due to the following effects such as energy depletion, node failures etc. As interference is less in relay communication the signal to interference ratio will be higher over long range communication. Higher throughput may be achieved by multi-hop transmission over direct communication if the numbers of hops are optimized.

\section{Proposed Relay Node Selection Method}

A novel method of selection of relay node is proposed here. The nodes that are being considered here are randomly distributed in a complex terrain and simplicity of calculation having uniform Sensitivity (S), which is defined as minimum Received Signal Strength $\left(\mathrm{RSS}_{\text {min }}\right)$ is necessary to generate adequate usable power output in presence of noise. The received signal strength at any node from the parent node will be affected due to propagation loss as a result of multipath fading and loss of packet information due to collision over multichannel interference. The variation of signal strength at any distant point due to the combined effect of propagation loss and multipath fading will have spatial and temporal variations. The nature of spatial variations of received signal strength is shown in Figure 1.

The continuous fluctuation of the signal strength may have ping-pong effect at the receiving node. To avoid ping-pong effect we select the relay node such that the 
average value of the received signal strength is little more than the sensitivity of the mobile node $S$. The value of the signal strength is proposed as Th which is $\Delta \mathrm{S}(0.03 \mathrm{dbm})$ above the sensitivity of the mobile node called threshold level as shown in Figure 1. If the received signal strength of any node is less than the threshold level then the particular node will not be considered as a relay node. Let $\mathrm{P}_{c}$ be the probability of collision due to multichannel interference, then $\left(1-\mathrm{P}_{c}\right)$ will be the probability of successful transmission $\left(\mathrm{P}_{s}\right)$ in a particular terrain.

From the above consideration, we find that for selection of suitable relay node, there are two important points to be considered: 1 . Received signal strength should be above the threshold that is $P_{r} \geq P_{h}$ and 2. Probability of collision should be minimum that is $P_{c} \leq x(0 \leq x \leq 1)$. We define a term $P_{e}$ as the effective received power for a successful reception at the particular node, which is product of $\mathrm{P}_{\mathrm{r}}$ and $\mathrm{P}_{\mathrm{s}}$.

That means $\left(1-P_{c}\right) * P_{r} \geq(1-x) * P_{h}$

where $P_{r} \geq P_{h} ; P_{c} \leq x$

The number of nodes in the ad hoc network between source and destination, which satisfy the above condition (shown in Equation (1)) may be considered as relay nodes for transmission. Now for a particular case where more than one node is satisfying the above condition, then the particular node is selected as first stage relay node where RSS is minimum (minimum received signal strength can optimize the hop distance) and if RSS value of two nodes is same then we select the relay node where $\left(1-\mathrm{P}_{c}\right)$ is maximum. Second phase relay node is selected considering

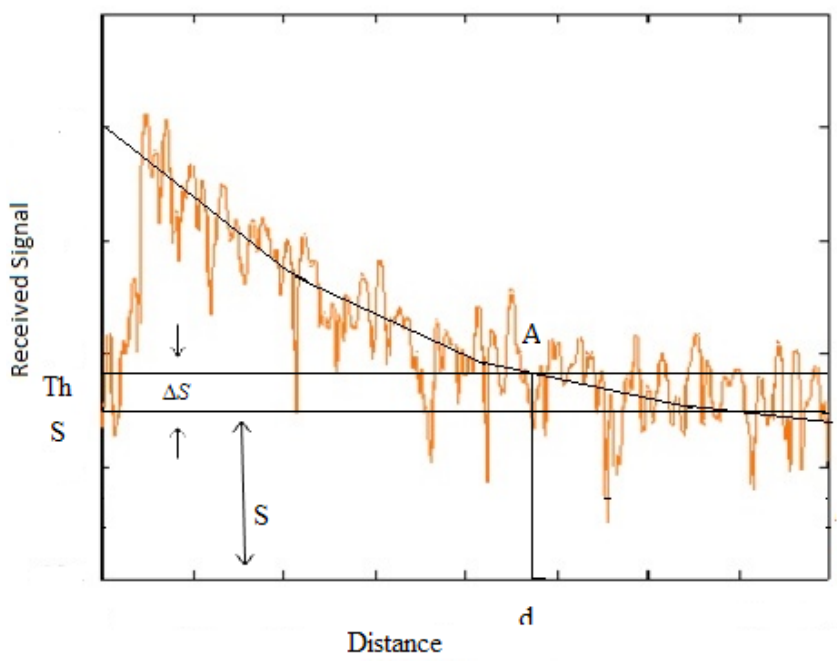

Figure 1. Variation of received signal strength. first phase relay node as a source node and the process of hopping through relay nodes will be continued till the information reaches the final destination node.

\subsection{Derivation of Propagation Loss}

Propagation loss $\left(\mathrm{P}_{\mathrm{L}}\right)$ for MIMO integrated MANET in different terrain has already been calculated $\frac{18-20}{2}$, as shown in subsequent section. Let, $\mathrm{PL}_{1-2}$ be the Propagation Loss between the nodes with multiple antennas which are placed in forest area, $\mathrm{PL}_{2-3}$ be the propagation loss between two nodes with multiple antennas which are in free space (outdoor propagation loss) propagation environment, $\mathrm{PL}_{3-4}$ represents combination of free space propagation loss and indoor propagation loss where one node is situated in outside the building and another one is inside the building, $\mathrm{PL}_{4-5}$ represents the indoor propagation loss where both the nodes are placed in inside the building and $\mathrm{PL}_{5-6}$ be the indoor propagation loss where two ad hoc nodes within the building but in different floor ${ }^{21}$. Thus propagation loss models are expressed as:

$$
P L_{1-2}=-10 \log _{10}\left(\frac{1}{M N} \sum_{f} \sum_{i=1}^{M} \sum_{j=1}^{N}\left\{\left[H_{i, j}\right]^{T} *[I]_{N^{* 1}}\right\} \times \frac{d^{4} d_{0}^{2}}{R^{2} f^{2}}\right)
$$

where d-forested depth in meter, R-radius of ad hoc nodes, $\mathrm{d}_{0}$-distance between two ad hoc nodes, f-frequency of the operating signal.

$$
P L_{2-3}=-10 \log _{10}\left(\frac{1}{M N} \sum_{f} \sum_{i=1}^{M} \sum_{j=1}^{N}\left\{\left[H_{i, j}\right]^{T} *[I]_{N^{* 1}}\right\} *\left(Q^{*} A_{r}\right)\right)
$$

$$
\left(Q * A_{r}\right)=\left(\frac{1}{2} \frac{e^{2}}{120 \pi}\right) *\left(\frac{\lambda^{2} g_{r}}{4 \pi l_{r}}\right)
$$

where e - Electric field strength

$$
\begin{aligned}
& P L_{3-4}=-10 \log _{10}\left(\frac{1}{M N} \sum_{f} \sum_{i=1}^{M} \sum_{j=1}^{N}\left\{\left[H_{i, j}\right]^{T} *[I]_{N^{* 1}}\right\} *\left(Q \times A_{r}\right)\right) \\
& +\left[-10 \log _{10}\left(\frac{1}{M N} \sum_{f} \sum_{i=1}^{M} \sum_{j=1}^{N}\left\{\left[H_{i, j}\right]^{T} *[I]_{N^{* 1}}\right\} *\left\{L_{f} F^{K_{1}} W^{K_{2}} R\right\}\right)\right]
\end{aligned}
$$

where F - Floor loss (For same floor the value of floor loss will be 2 to 3 ), $\mathrm{W}$ - Wall loss, $\mathrm{R}$ - Reflection loss, $\mathrm{K}_{1}$ Number of floor, $\mathrm{K}_{2}$ - Number of wall

$$
P L_{4-5}=-10 \log _{10}\left(\frac{1}{M N} \sum_{f} \sum_{i=1}^{M} \sum_{j=1}^{N}\left\{\left[H_{i, j}\right]^{T} *[I]_{N^{* 1}}\right\} \times\left\{L_{f} F^{K_{1}} W^{K_{2}} R\right\}\right)
$$




$$
P L_{5-6}=\left[-10 \log _{10}\left(\frac{1}{M N} \sum_{f} \sum_{i=1}^{M} \sum_{j=1}^{N}\left\{\left[H_{i, j}\right]^{T} *[I]_{N^{* 1}}\right\} \times\left\{L_{f} F^{K_{1}} W^{K_{2}} R\right\}\right)\right]
$$

If propagation loss is subtracted from transmitted signal the effective received signal strength can be easily estimated. Now, to calculate $\mathrm{P}_{\mathrm{e}}$ we need to find out probability of collision $\left(\mathrm{P}_{\mathrm{C}}\right)$ and hence proceed to selection of relay node.

\subsection{Derivation of Probability of Successful Transmission}

Let us consider a model of transmission process via relay node is shown in Figure 2. In the model diagram source node $S$ transmit data to destination node $\mathrm{D}$ via relay node $\mathrm{R} . \mathrm{h}_{\mathrm{sr}}, \mathrm{h}_{\mathrm{sd}}, \mathrm{h}_{\mathrm{rd}}$ are the channel gain of the source to relay, source to destination and relay to the destination node, $P_{S}^{\prime}$ is transmit signal power and $P_{r}^{\prime}$ is received signal power. We know that the transmission probability of any channel depends on channel capacity of the channel. Channel capacity of any channel is the maximum value of mutual information. So, to estimate the probability of transmission the first action needed is to calculate mutual information of the channel. Mutual information of cooperative communication in amplify-and-forward method is (Zhang et al. 2009) found as:

$$
I_{S D}^{A F}=\log \left(1+P_{s}^{\prime}\left|h_{s d}\right|^{2}+\frac{P_{s}^{\prime}\left|h_{s r}\right|^{2} P_{r}^{\prime}\left|h_{r d}\right|^{2}}{1+P_{s}^{\prime}\left|h_{s r}\right|^{2}+P_{r}^{\prime}\left|h_{r d}\right|^{2}}\right)
$$

In our work, we consider MIMO integrated mobile ad hoc network, so it is needed to estimate mutual information of MIMO channel. Mutual information of amplify-andforward method for MIMO channel is calculated (in appendix I for multiple numbers of channels).

$$
\left(I_{S D}^{A F}\right)_{M I M O}=\log \left(1+P_{s}^{\prime} \sum_{i} \sum_{j}\left|h_{s d}^{i j}\right|^{2}+\frac{P_{s}^{\prime} \sum_{i} \sum_{j}\left|h_{s r}^{i j}\right|^{2} P_{r}^{\prime} \sum_{i} \sum_{j}\left|h_{r d}^{i j}\right|^{2}}{1+P_{s}^{\prime} \sum_{i} \sum_{j}\left|h_{s r}^{i j}\right|^{2}+P_{r}^{\prime} \sum_{i} \sum_{j}\left|h_{r d}^{i j}\right|^{2}}\right)
$$

where $i(1,2, . . M)$ and $j(1,2, \ldots N)$ signify a number of transmitting and receiving antennas. Channel capacity of the MIMO channel using amplify-and-forward method is

$$
\left(C_{A F}\right)_{M I M O}=\max \left(I_{S D}^{A F}\right) \text {. }
$$

To estimate probability density function we need to study the SNIR of the received signal as CDF function that depends on SNIR. SNIR of the threshold value $(\beta)$ of any channel is found by inverting the capacity function $\beta \propto C^{-1} . \beta$ is used to generate Cumulative Distributive Function $(\mathrm{CDF})^{22}$. From this, the estimation of Probability Density Function (PDF) of the received signal is done which is expressed as:

$$
f_{y_{i}}(y)=\left(1-p_{i}\right) \delta(y)+p_{i}\left(\frac{n_{i}}{\omega_{i}}\right)^{n_{i}} \frac{1}{\Gamma\left(n_{i}\right)} y^{n_{i}-1} e^{-y^{n_{i}} / \omega_{i}} u(y)
$$

where $\mathrm{p}_{\mathrm{i}}$ is the active probability of a particular noden $\mathrm{i}_{\mathrm{i}}$. Signal at receiving end $\mathrm{y}=\left[y_{1}, y_{2}, y_{3}, \ldots y_{n}\right]$

Probability of successful transmission for throughout the network consisting n number of nodes:

$$
P_{S}=\int_{0}^{n} f_{y_{i}}(y) d y
$$

Probability of collision $P_{c}=1-P_{s}$

Intermediate relay node estimated on basis of propagation loss and the probability of successful transmission.

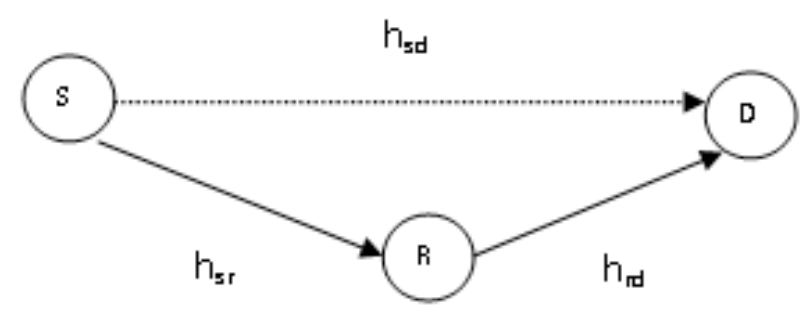

Figure 2. Example network for cooperative transmission.

\subsection{The Steps Followed for the Selection of Relay Nodes are as Follows}

Step 1: Estimating received signal strength $\left(\mathrm{P}_{\mathrm{r}}\right)$ and probability of successful transmission $\left(\mathrm{P}_{\mathrm{s}}\right)$.

Step 2: Define a threshold value $\left(\mathrm{P}_{\text {th }}\right)$ slightly just above sensitivity of the node and probability of collision ( $\mathrm{x}$ ) where $0 \leq x \leq 1$.

Step 3: Check the condition for selection of relay node as, (effective received signal strength)

$$
P_{s} * P_{r} \geq(1-x) * P_{t h}
$$

Step 4: If this condition is satisfied then the node is considered as relay node where effective received signal strength 
is minimum. For same value of minimum effective received signal strength, the particular node is selected as relay node where probability of successful transmission is maximum. If more than one node meets all the above condition in some cases, then any one of the nodes can be selected as a relay node.

\section{Proposed MPCR Algorithm Supporting the Relay Node Selection Method}

In the proposed (MPCR) algorithm, the best partner (suitable relay node) selection routing (optimized path) is decided on the basis of received power $\left(\mathrm{P}_{\mathrm{r}}\right)$ by the node (estimated in section 3.i) and probability of successful transmission $\left(\mathrm{P}_{\mathrm{s}}\right)$ (estimated in section 3.2). In the model network shown in Figure 3, 20 numbers of MIMO integrated ad hoc nodes are randomly distributed in a combined terrain (forest, free space and indoor environment). Minimum power consumed route is selected at the time of transmission throughout all the nodes of the network. Any ad hoc node first broadcast the route update to its neighbours periodically. Each neighbouring node that receives the updates for the first time rebroadcasts the update and marks the node from which it receives recursively till every node in the network has rebroadcast the update once and finds its parent. In this way, the routing table for effective power $\left(\mathrm{P}_{\mathrm{e}}\right)$, multiplication of received power $\left(\mathrm{P}_{\mathrm{r}}\right)$ by the node and probability of successful transmission $\left(\mathrm{P}_{\mathrm{s}}\right)$, is updated in a periodic way. From this routing table of the source node, we compare the total power of the entire surrounding node within its transmission range. Finally the best relay partner node is selected where total loss is minimum. In this way, first phase relay node selection is done and then this node becomes the source node for selection of next phase relay selection. Again the path loss is compared in the same way except at the parent node (from where the source receives the data) and the next relay node is selected with minimum loss route. This process repeats and updates the routing table until it reaches the destination. In the above Figure, source node 1 transmits the packet to its neighbours within its transmission range. Node 4, 6 and 16 are within the transmission range of source node 1 . The source node floods the data to its neighbour's nodes and checks which node satisfies the condition for Equation 1. If any node does not satisfy the condition then this node is not con- sidered for the relay node selection process. In this case as node 4, 6 and 16 satisfy the condition of Equation 1 they can be considered as relay nodes. Among all these nodes, effective received power $\left(\mathrm{P}_{\mathrm{e}}\right)$ is same of the nodes 6 and 16 , less than that for node 4 . So, in this case node 6 and 16 can be selected as relay node. Among these two nodes the particular node is considered as a relay node where the probability of successful transmission is maximum (node 16 satisfy all these conditions), here node 16 is selected as first stage relay node. In the second stage node 16 acts as a source node and transmit signal to all the nodes 3, 5 and 14 which are within transmission range but except its parent node 1 to find the second phase relay node. The process continues until transmitted signal reaches the final destination node 20. In this algorithm, the parent node of each transmission will not be considered as receiving node so that back propagation is prohibited. The flowchart of the algorithm is shown in Figure 4.

\section{Simulation Result and Discussion}

This section presents the simulation results in MATLAB environment to validate the proposed routing algorithm. In this paper, a model network with 20 numbers of nodes has been considered in different terrain (forest, free space and indoor). The propagation loss and packet loss due to the probability of successful transmission are calculated for all nodes in different terrain. The route using MPCR algorithm is shown in Figure 5. The best route is: node1$>$ node16->node3->node12->node9->node15->node20. On basis of some performance metrics a comparative study is done for MPCR protocol with some other existing routing protocol. Figure 6 shows the maximum through-

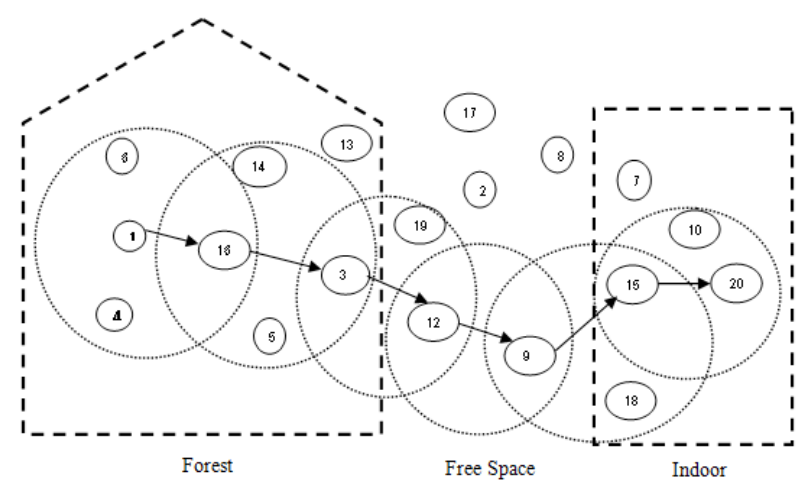

Figure 3. Proposed network for verification of algorithm. 


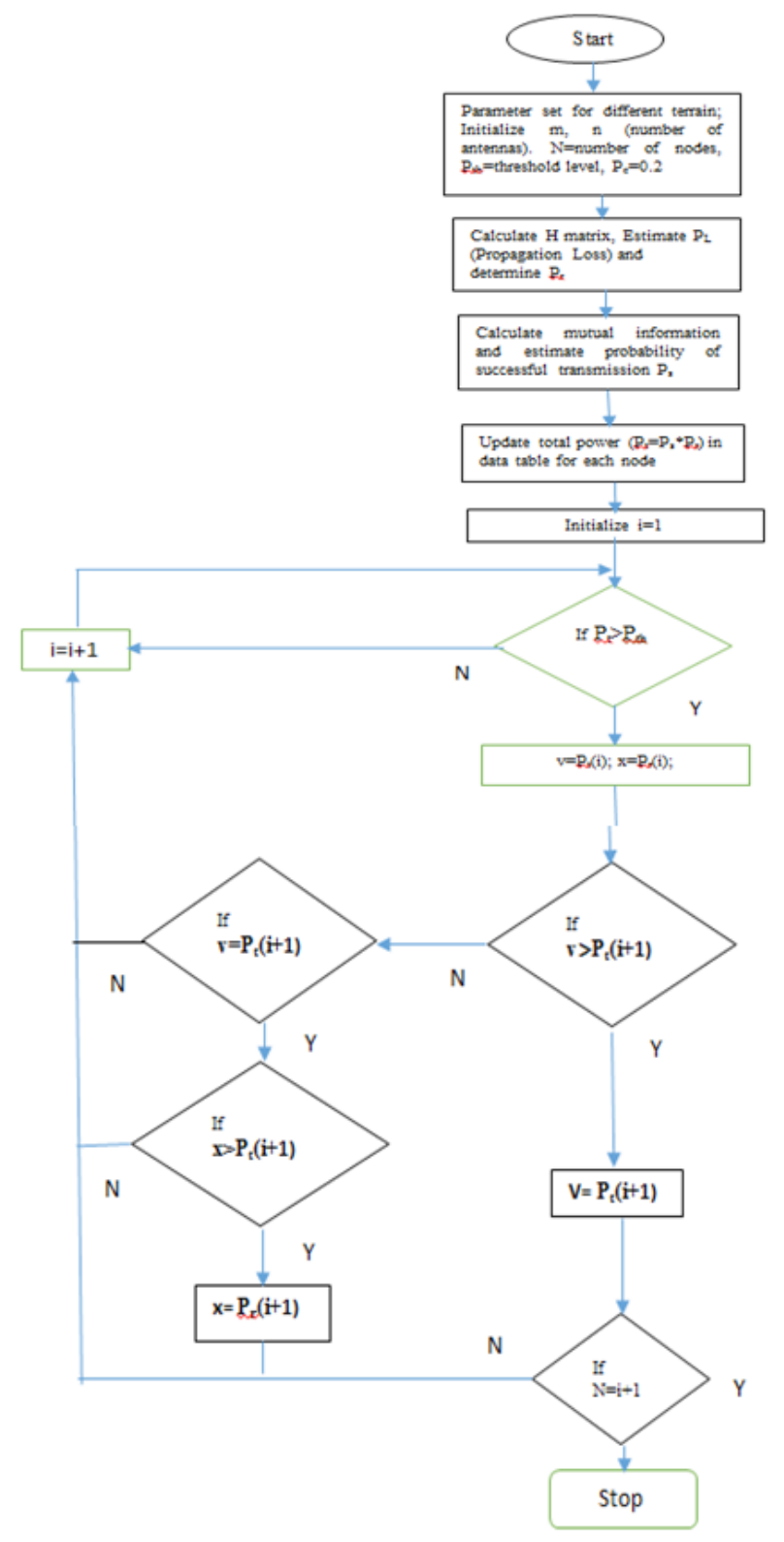

Figure 4. Flowchart of the proposed algorithm.

put for this proposed algorithm and it is observed from Figure 7 that the number of dead node is almost zero for this algorithm. Figure 8 depicts a comparative study of this proposed algorithm with other existing algorithm. It is also found that the transmission delay is reduces for this algorithm which makes the transmission faster.

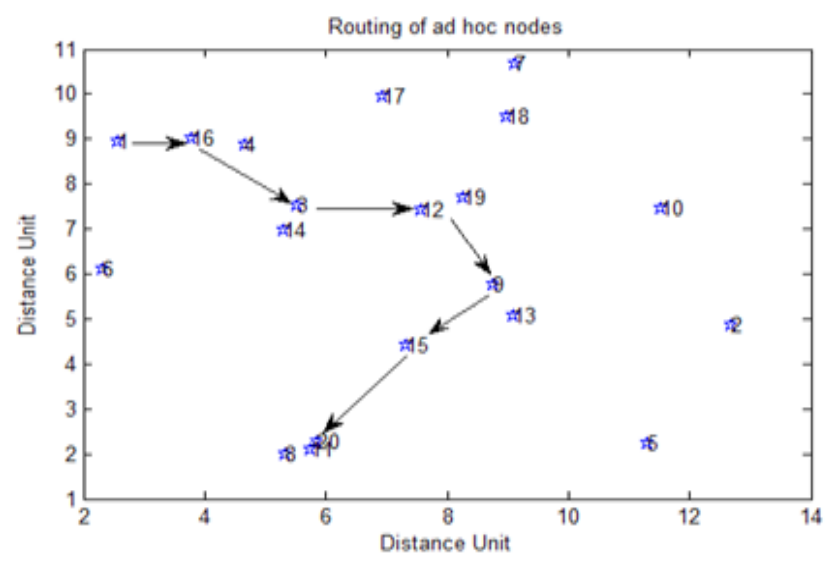

Figure 5. Simulated route of the network.

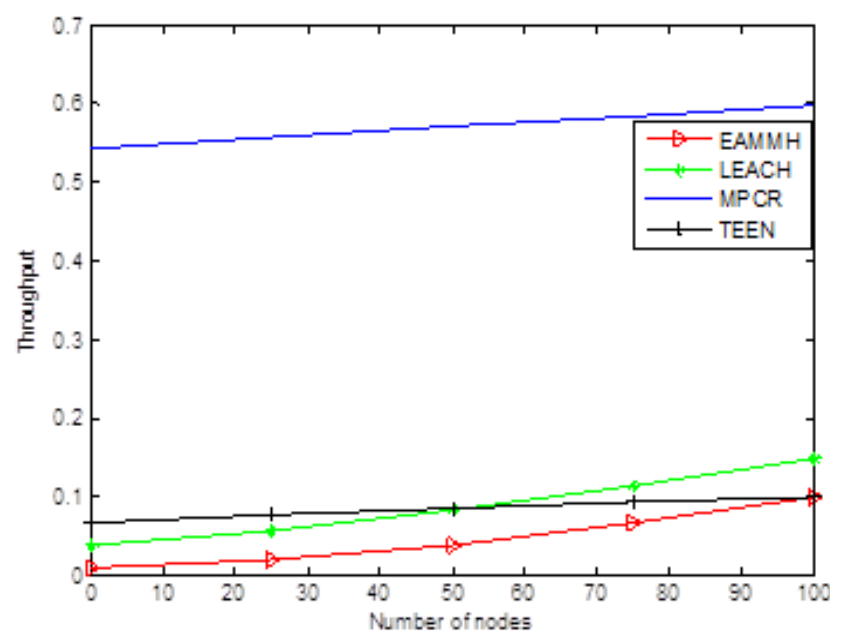

Figure 6. Throughput of the network.

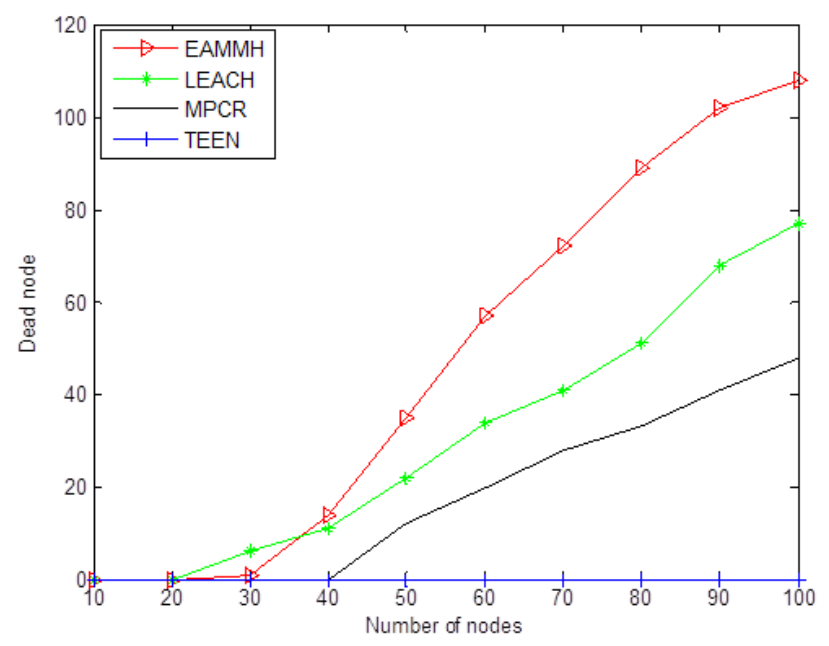

Figure 7. Dead node of the network. 


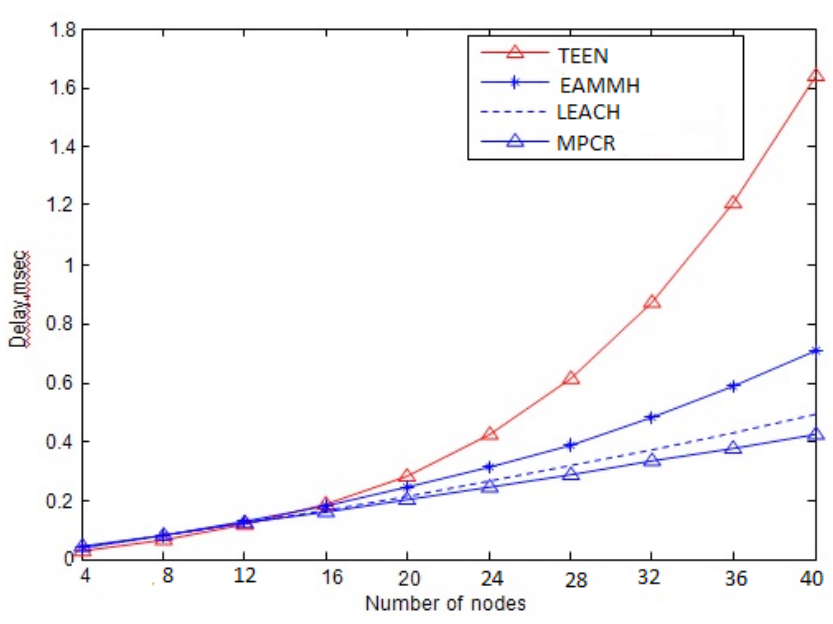

Figure 8. Nodes vs. transmission delay at different broadcast algorithm.

\section{Conclusions}

MPCR algorithm provides seamless communication as the Signal to Noise Interference Ratio is much more due to short range communication. Study of performance of the proposed algorithm provides higher transmission rate multi-hop transmission over direct communication. The proposed MPCR algorithm is based on the cooperative transmission. Cooperation of transmission is done by selecting best suitable relay node. The relay node is selected on basis of effecting received signal strength of mobile ad hoc node and probability of successful transmission. Consideration of the relay node makes short distance communication which can improve the SNR. In this work, we mainly focus on minimizing energy consumption and maximizing network performance. From the performance analysis it may be shown that proposed algorithm works very efficiently in hybrid terrain. The proposed algorithm may be noted that out-performs most of the existing algorithm presently in use which is presented in the simulated result.

\section{Reference}

1. Liu H, Jai X, Wan J, Liu X, Yao F. A distributed and efficient flooding scheme using 1-hop information in Mobile Ad Hoc Networks. IEEE Transactions on Parallel and Distributed Systems. 2007; 18(5):658-71. Crossref.

2. Mohammad A, Mikki W. Energy efficient location aided routing protocol for wireless MANETs. International
Journal of Computer Science and Information Security. 2009; 4(1):1-2.

3. Shah S, Islam S. A survey on cooperative communication in wireless networks. International Journal of Intelligent Systems and Applications. 2014; 7(1):66-78. Crossref.

4. Nosratinia A, Hedayat A. Cooperative communication in wireless networks. IEEE International Symposium on Personal Indoor and Mobile Radio Communications; 2013. p. $2275-80$.

5. Manickam P, Guru Baskar P, Girija M, Manimegalai D. Performance comparisons of routing protocols in Mobile Ad Hoc Networks. International Journal of Wireless and Mobile Networks. 2011; 3(1):98-106. Crossref.

6. Taksande V, Kulat D. Performance comparison of DSDV, DSR, AODV protocol with IEEE 802.11MAC for chain topology for a Mobile Ad-Hoc Network using NS-2. Proceedings of the 2nd National Conference-Computing, Communication and Sensor Network (CCSN '11); 2011. p. 26-31.

7. Doan $\mathrm{H}$, Thuan D. A method for finding the appropriate number of clusters. International Arab Journal of Inform Tech. 2017; 15(3):1-8.

8. Vats K, Sachdeva M, Saluja K, Rathee A. Simulation and performance analysis of OLSR routing protocol using OPNET. International Journal of Advanced Research in Computer Science and Software Engineering. 2012; 2(2):1-4.

9. Karthik S, Kannan S, Valarmathi M, Arunachalam V, Ravichandran T. A performance analysis and comparison of multi-hop wireless ad-hoc network routing protocols in MANET. International Journal of Academic Research. 2012; 2(4):36-41.

10. Perumal M, Dhandapani S. Modeling and simulation of a novel relay node based secure routing protocol using multiple mobile sinks for Wireless Sensor Networks. The Scientific World Journal. 2015; 495945:1-9. Crossref.

11. Hosseinzadeh H, Razzazi F. An SNR unaware large margin automatic modulations classifier in variable SNR environments. International Arab Journal of Inform Tech. 2017; 15(3):1-8.

12. Kaur S. Performance evaluation of hybrid routing protocols in Mobile Ad Hoc Network. International Journal of Advanced Research in Computer Science and Software Engineering. 2013; 3(9):634-40.

13. Raheja. K, Maakar S. A survey on different hybrid routing protocols of MANET. International Journal of Computer Science and Information Technologies. 2014; 5(4):5512-16.

14. Xu S, Wang F. Analysis and comparison of relay node selection algorithm of cooperative communication. Information Technology Journal. 2013; 12(3):434-8. Crossref.

15. Zhang B, Diao X, Ding K, Yan H. A physical topology discovery method based on AFTs of down constraint. International Arab Journal of Inform Tech. 2017; 15(1):2-9. 
16. Hasan C, Altman E, Gorce J. Partner selection for decodeand-forward cooperative relaying: A matching theoretic approach. IEEE 24th Annual International Symposium on Personal, Indoor and Mobile Radio Communications; 2013. p. 2275-80. Crossref.

17. Moraes H, Adjadpour J. Throughput-delay analysis of Mobile Ad Hoc Networks with a multi-copy relaying strategy. First Annual IEEE Communications Society Conference on Sensor and Ad Hoc Communications and Networks; 2004.

18. Adegoke AS, Siddle D. Investigation into vegetation effects on propagation effects on propagating radio waves. 3rd Conference on Applied Radio Systems Research and Smart Wireless Communications; 2012.

19. Meng YS, Yee HL, Boon Chong Ng. Empirical near ground path loss modelling in a forest at VHF and UHF bands. 2009 IEEE Transactions on Antennas and Propagation; 2009; 57(5):1461-8. Crossref.

20. Andersen JB, Rappaport TS, Yoshida S. Propagation measurements and models for wireless communications channels. IEEE Communications Magazine. 1995; 33(1):42-9. Crossref.

21. Chowdhuri S, Chaudhury S, Banerjee P. Propagation analysis of hybrid model of MIMO ad hoc network and implement less propagation loss algorithm to find the minimum loss route. International Journal of Information and Communication Technology. 2016; 10(1):66-80. Crossref.

22. Torrieri D, Valenti M. The outage probability of a finite ad hoc network in Nagagami fading. IEEE Communication Society. 2012; 1207.711:1-9.

\section{Appendix I:}

$$
\begin{aligned}
& \ln \operatorname{det}\left[\begin{array}{cc}
1+\beta_{11} & \beta_{12} \\
\beta_{21} & 1+\beta_{22}
\end{array}\right]=\text { In } \\
& {\left[1+\left(\beta_{11}+\beta_{22}\right)+\left(\beta_{11} \beta_{22}-\beta_{21} \beta_{12}\right)\right]} \\
& |H|^{2}=H^{+} H \\
& H=\left[\begin{array}{ll}
h_{11} & h_{12} \\
h_{21} & h_{22}
\end{array}\right] \quad H^{+}=\left[\begin{array}{ll}
h_{11} & h_{21} \\
h_{12} & h_{22}
\end{array}\right] \\
& 1+H H^{H}=1+\left[\begin{array}{ll}
h_{11} & h_{12} \\
h_{21} & h_{22}
\end{array}\right]\left[\begin{array}{ll}
h_{11} & h_{21} \\
h_{12} & h_{22}
\end{array}\right] \\
& \operatorname{det}\left(1+H H^{H}\right)=\left(\begin{array}{cc}
1+h_{11}^{2}+h_{12}^{2} & h_{11} h_{21}+h_{12} h_{22} \\
h_{21} h_{11}+h_{22} h_{12} & 1+h_{21}^{2}+h_{22}^{2}
\end{array}\right) \\
& \left(1+h_{11}^{2}+h_{12}^{2}\right)\left(1+h_{21}^{2}+h_{22}^{2}\right)-\left(h_{11} h_{21}+h_{12} h_{22}\right)\left(h_{21} h_{11}+h_{22} h_{12}\right) \\
& =1+h_{11}^{2}+h_{22}^{2}+h_{12}^{2}+h_{21}^{2}+\left(\begin{array}{ll}
\left.h_{11} h_{22}-h_{21} h_{12}\right) \\
=
\end{array}\right)
\end{aligned}
$$

